\title{
Adaptive Chaos Synchronization Control of Nonlinear PMSM System Using Extended State Observer
}

\author{
Zijing Cheng, ${ }^{1}$ Guangyue Xue, ${ }^{1}$ Chong Wang, ${ }^{1}$ and Qiang Chen ${ }^{2}$ \\ ${ }^{1}$ Beijing Institute of Satellite Information Engineering, Beijing 100000, China \\ ${ }^{2}$ College of Information Engineering, Zhejiang University of Technology, Hangzhou 310023, China \\ Correspondence should be addressed to Qiang Chen; sdnjchq@zjut.edu.cn
}

Received 12 July 2016; Revised 9 October 2016; Accepted 19 October 2016

Academic Editor: Uchechukwu E. Vincent

Copyright (c) 2016 Zijing Cheng et al. This is an open access article distributed under the Creative Commons Attribution License, which permits unrestricted use, distribution, and reproduction in any medium, provided the original work is properly cited.

\begin{abstract}
This paper proposes an adaptive chaos synchronization control scheme for nonlinear permanent magnet synchronous motor (PMSM) systems by using extended state observer (ESO). Frist of all, a chaotic PMSM system is built through an affine transformation and a time scale transformation of the mathematical PMSM model. Then, an adaptive sliding mode controller is developed based on the extended state observer to achieve the synchronization performance of two chaotic PMSM systems. Moreover, an adaptive parameter law of the control gain is designed to reduce the chattering problem existing in the traditional sliding mode control. Finally, the effectiveness of the proposed method is verified by simulation results.
\end{abstract}

\section{Introduction}

The research on chaos control and synchronization has been widely studied since the chaos phenomenon was discovered [1-4]. As far as we know, chaos problem has been proved to exist in various practical systems. In the engineering field, chaos may cause irregular operation and affect the stability of the motors. Consequently, how to deal with the chaos problem in motors is still a significant problem.

Lots of control and synchronization schemes have been represented since the discovery of chaotic impact on the motor control performance, such as finite-time control $[5,6]$, unidirectional correlation control [7], sliding mode control [8-11], linear control [12], dynamic surface control [13, 14], optimal control [15], and neural network control [16]. In [5], an adaptive finite-time control method is proposed for PMSM system to suppress the chaos behavior with parameter uncertainties. The uncertain parameters which are caused by external factors can be solved and the chaos in motor can be effectively stabilized. Reference [14] presents a dynamic surface control method based on neural network (NN) for PMSM system. The NN is adopted to approximate the system nonlinearities like disturbance and unknown parameters, and the control performance is guaranteed by using the designed control method.

Most of the methods mentioned above can effectively eliminate the influence of chaos in PMSM, but the robustness of the system may be not guaranteed when the system has different initial conditions or disturbances. Due to the strong robustness and antidisturbance ability, sliding mode control (SMC) has been widely applied to the chaotic control in PMSM systems. In [8], a sliding mode control based on fuzzy neural network is investigated for the chaotic PMSM to suppress the chaos and improve the tracking control performance. Reference [9] proposes a high robust controller based on the traditional sliding mode control for PMSM with interference and uncertainties. The system has a good control performance and robustness by using the proposed method. But unfortunately, the robustness of SMC usually has a strong dependence on the control gain, and the control performance may become worse when the disturbance or uncertainties of the system are beyond of the control range. Consequently, in this paper, an adaptive law of the control gain is designed to solve this problem, and the extended state observer is employed to estimate the system uncertainties and unknown parameters. For the dual-motor cooperative control system 
with chaos, the adaptive sliding mode control is investigated to guarantee the synchronization control performance, and the chattering problem in traditional sliding mode control is also improved.

The rest of this paper is organized as follows. The chaotic PMSM model and the extended state observer are derived in Sections 2 and 3, respectively. In Section 4, an adaptive sliding mode control scheme is developed for chaotic PMSM system and the system analysis is provided. Section 5 provides the simulation results and the conclusion is given by Section 6 .

\section{System Description}

The mathematical model of a nonlinear PMSM system is expressed as

$$
\begin{aligned}
& \frac{d i_{d}}{d t}=\frac{\left(-R_{s} i_{d}+n_{p} L_{q} \omega_{m} i_{q}+u_{d}\right)}{L_{d}} \\
& \frac{d i_{q}}{d t}=\frac{\left(-R_{s} i_{q}-n_{p} L_{d} \omega_{m} i_{d}-n_{p} \psi_{f} \omega_{m}+u_{q}\right)}{L_{q}} \\
& \frac{d \omega_{m}}{d t}=\frac{\left\{n_{p}\left[\psi_{f} i_{q}+\left(L_{d}-L_{q}\right) i_{d} i_{q}\right]-T_{L}-B \omega_{m}\right\}}{J}
\end{aligned}
$$

where $i_{d}, i_{q}$ are the components of stator current in $d$-axis and $q$-axis; $u_{d}, u_{q}$ are the components of stator voltage in $d$-axis and $q$-axis; $L_{d}, L_{q}$ are the equivalent inductances of stator windings in $d$-axis and $q$-axis; $\psi_{f}$ is the flux which is generated by the permanent magnet; $R_{s}$ is the stator resistance; $T_{L}$ denotes the load torque; $J$ is the rotary inertia; $B$ is the coefficient of friction; $n_{p}$ represents the pole pairs of motor; $\omega_{m}$ is the mechanical angular velocity of rotor.

Define

$$
\begin{aligned}
x & =\left[\begin{array}{lll}
i_{d} & i_{q} & \omega_{m}
\end{array}\right], \\
\tilde{x} & =\left[\begin{array}{lll}
\widetilde{i}_{d} & \widetilde{i}_{q} & \widetilde{\omega}_{m}
\end{array}\right], \\
b & =\frac{L_{q}}{L_{d}} \\
k & =\frac{\beta}{\left(n_{p} \tau \psi_{f}\right)} \\
\tau & =\frac{L_{d}}{R_{s}}, \\
\lambda & =\left[\begin{array}{ccc}
\lambda_{d} & 0 & 0 \\
0 & \lambda_{q} & 0 \\
0 & 0 & \lambda_{\omega}
\end{array}\right]=\left[\begin{array}{ccc}
b k & 0 & 0 \\
0 & k & 0 \\
0 & 0 & \frac{1}{\tau}
\end{array}\right],
\end{aligned}
$$

and choose the affine transformation $x=\lambda \tilde{x}$ and time scale transformation $t=\tau \tilde{t}$ for system (1); then (1) can be transformed into

$$
\begin{aligned}
\frac{d \tilde{i}_{d}}{d t} & =-\widetilde{i}_{d}+\widetilde{\omega}_{m} \widetilde{i}_{q}+\widetilde{u}_{d} \\
\frac{d \tilde{i}_{q}}{d t} & =-\widetilde{i}_{q}-\widetilde{\omega}_{m} \widetilde{i}_{d}+\gamma \widetilde{\omega}_{m}+\widetilde{u}_{q} \\
\frac{d \widetilde{\omega}_{m}}{d t} & =\sigma\left(\widetilde{i}_{q}-\widetilde{\omega}_{m}\right)+\varepsilon \widetilde{i}_{d} \widetilde{i}_{q}-\widetilde{T}_{L},
\end{aligned}
$$

where $\gamma=-\psi_{f} / k L_{q}, \sigma=\beta \tau / J, \varepsilon=\left(n_{p} b \tau^{2} k^{2}\left(L_{d}-L_{q}\right)\right) / J$, $\tilde{u}_{d}=(1 / k R) u_{d}, \tilde{u}_{q}=(1 / k R) u_{q}$, and $\widetilde{T}_{L}=\left(\tau^{2} / J\right) T_{L}$. Since the conversion is linear transformation, it does not change the characteristics of the system. The purpose of the transformation is to simplify system (1) and to obtain the corresponding chaotic model. For the uniform air gap, we have $L_{d}=L_{q}=L$, and then (3) can be rewritten as

$$
\begin{aligned}
\frac{d \tilde{i}_{d}}{d t} & =-\tilde{i}_{d}+\widetilde{\omega}_{m} \tilde{i}_{q}+\widetilde{u}_{d} \\
\frac{d \tilde{i}_{q}}{d t} & =-\widetilde{i}_{q}-\widetilde{\omega}_{m} \tilde{i}_{d}+\gamma \widetilde{\omega}_{m}+\widetilde{u}_{q} \\
\frac{d \widetilde{\omega}_{m}}{d t} & =\sigma\left(\tilde{i}_{q}-\widetilde{\omega}_{m}\right)-\widetilde{T}_{L},
\end{aligned}
$$

where $\tilde{i}_{d}, \widetilde{i}_{q}$, and $\widetilde{\omega}_{m}$ are the state variables, which represent the stator currents of direct axis and quadrature axis and the angular frequency of rotor, respectively; $\widetilde{u}_{d}$ and $\widetilde{u}_{q}$ denote the stator voltages of the direct axis and quadrature axis, respectively; $\widetilde{T}_{L}$ is the external torque; $\sigma, \gamma$ are the constant parameters. The external torque is set as $\widetilde{T}_{L}=0$ when $\widetilde{u}_{d}=0$ and $\widetilde{u}_{q}=0$. Then, we can obtain the following chaotic PMSM model:

$$
\begin{aligned}
\frac{d \widetilde{i}_{d}}{d t} & =-\widetilde{i}_{d}+\widetilde{\omega}_{m} \widetilde{i}_{q} \\
\frac{d \tilde{i}_{q}}{d t} & =-\widetilde{i}_{q}-\widetilde{\omega}_{m} \widetilde{i}_{d}+\gamma \widetilde{\omega}_{m} \\
\frac{d \widetilde{\omega}_{m}}{d t} & =\sigma\left(\widetilde{i}_{q}-\widetilde{\omega}_{m}\right) .
\end{aligned}
$$
have

Define $x_{1}=\widetilde{\omega}_{m}, x_{2}=\widetilde{i}_{q}$, and $x_{3}=\widetilde{i}_{d}$, and from (5) we

$$
\begin{aligned}
& \dot{x}_{1}=\sigma\left(x_{2}-x_{1}\right) \\
& \dot{x}_{2}=\gamma x_{1}-x_{1} x_{3}-x_{2} \\
& \dot{x}_{3}=x_{1} x_{2}-x_{3},
\end{aligned}
$$


where $x_{1}, x_{2}$, and $x_{3}$ are the states. The PMSM system (6) is regarded as the active system, and the corresponding passive PMSM system is given by

$$
\begin{aligned}
& \dot{y}_{1}=\sigma\left(y_{2}-y_{1}\right) \\
& \dot{y}_{2}=\gamma y_{1}-y_{1} y_{3}-y_{2}+u \\
& \dot{y}_{3}=y_{1} y_{2}-y_{3},
\end{aligned}
$$

where $y_{1}, y_{2}$, and $y_{3}$ are the states and $u$ is the system controller.

The objective of this paper is to design the controller $u$ such that the synchronization performance of the states $x_{i}$ and $y_{i}, i=1,2,3$ between chaotic systems (6) and (7) are achieved.

\section{Extend State Observer Design}

Define the synchronization errors as $e_{1}=y_{1}-x_{1}, e_{2}=y_{2}-x_{2}$, and $e_{3}=y_{3}-x_{3}$, and we can obtain the following error system:

$$
\begin{aligned}
& \dot{e}_{1}=\sigma\left(e_{2}-e_{1}\right) \\
& \dot{e}_{2}=\gamma e_{1}-y_{1} y_{3}+x_{1} x_{3}-e_{2}+u \\
& \dot{e}_{3}=y_{1} y_{2}-x_{1} x_{2}-e_{3} .
\end{aligned}
$$

In (8), we have the following fact:

$$
\begin{aligned}
& y_{1} y_{3}-x_{1} x_{3}=-e_{1} e_{3}+e_{1} y_{3}+e_{3} y_{1} \\
& y_{1} y_{2}-x_{1} x_{2}=-e_{1} e_{2}+e_{1} y_{2}+e_{2} y_{1} .
\end{aligned}
$$

Substituting (9) into (8) yields

$$
\begin{aligned}
& \dot{e}_{1}=\sigma\left(e_{2}-e_{1}\right) \\
& \dot{e}_{2}=\gamma e_{1}+e_{1} e_{3}-e_{1} y_{3}-e_{3} y_{1}-e_{2}+u \\
& \dot{e}_{3}=-e_{1} e_{2}+e_{1} y_{2}+e_{2} y_{1}-e_{3} .
\end{aligned}
$$

Then, error system (10) can be divided into the following two subsystems:

$$
\begin{aligned}
& \dot{e}_{1}=\sigma\left(e_{2}-e_{1}\right) \\
& \dot{e}_{2}=\gamma e_{1}+e_{1} e_{3}-e_{1} y_{3}-e_{3} y_{1}-e_{2}+u, \\
& \dot{e}_{3}=-e_{1} e_{2}+e_{1} y_{2}+e_{2} y_{1}-e_{3} .
\end{aligned}
$$

Remark 1 . From (12), it can be easily concluded that $\dot{e}_{3}=-e_{3}$ when $e_{1}$ and $e_{2}$ converge to zero, which leads $e_{3}$ to converge to zero finally. That means the error $e_{3}$ acts as the interior dynamics of whole system (10). Therefore, the control task is transferred to design the controller $u$ for subsystem (11) and guarantee the convergence of $e_{1}$ and $e_{2}$.

Define $g_{1}=e_{1}, g_{2}=\sigma\left(e_{2}-e_{1}\right)$, and then subsystem (11) can be transformed into the following Brunovsky form:

$$
\begin{aligned}
& \dot{g}_{1}=g_{2} \\
& \dot{g}_{2}=a(e)+b u,
\end{aligned}
$$

where $a(e)=\sigma\left[\gamma e_{1}+e_{1} e_{3}-e_{3} y_{1}-e_{1} y_{3}-e_{2}-\sigma\left(e_{2}-e_{1}\right)\right]$, $b=\sigma$.

In order to facilitate the design of controller $u$, the system uncertainty $a(e)$ and unknown parameter $b$ in (13) should be measured by designing an observer. Define $a_{0}=a(e)+\Delta b u$, $\Delta b=b-b_{0}$, where $b_{0}$ is the estimation of $b$ and can be given by the prior experience directly. Then, designing an extended state $g_{3}=a_{0}$, system (13) can be transformed into

$$
\begin{aligned}
& \dot{g}_{1}=g_{2} \\
& \dot{g}_{2}=g_{3}+b_{0} u \\
& \dot{g}_{3}=\dot{a}_{0} .
\end{aligned}
$$

Define $z_{i}, i=1,2,3$, as the observation values of the states $g_{i}$ in (14), and the corresponding observer errors are given as $e_{0 i}=z_{i}-g_{i}$; then the nonlinear extended state observer is expressed as

$$
\begin{aligned}
& \dot{z}_{1}=z_{2}-\beta_{1} e_{01} \\
& \dot{z}_{2}=z_{3}-\beta_{2} \text { fal }\left(e_{01}, \alpha_{1}, \delta\right)+b_{0} u \\
& \dot{z}_{3}=-\beta_{3} \operatorname{fal}\left(e_{01}, \alpha_{2}, \delta\right)
\end{aligned}
$$

where $\beta_{1}, \beta_{2}, \beta_{3}>0$ are the observer tuning gains; $\mathrm{fal}(\cdot)$ is a nonlinear continuous function with the following form:

$$
\mathrm{fal}\left(e_{01}, \alpha_{i}, \delta\right)= \begin{cases}\frac{e_{01}}{\delta^{1-\alpha_{i}}} & \left|e_{01}\right| \leq \delta \\ \left|e_{01}\right|^{\alpha_{i}} \operatorname{sign}\left(e_{01}\right) & \left|e_{01}\right|>\delta\end{cases}
$$

where $\delta>0$ denotes the interval length of the linear segment; $0<\alpha_{i}<1$ is a constant.

\section{Controller Design and Stability Analysis}

4.1. Controller Design. In order to stabilize the system tracking errors $e_{1}$ and $e_{2}$ to the zero, an adaptive controller $u$ is designed in this subsection based on the sliding mode control technique.

The sliding mode surface is designed as

$$
s=g_{2}+\lambda_{1} g_{1}
$$

Differentiate $s$, and we can obtain

$$
\dot{s}=\dot{g}_{2}+\lambda_{1} \dot{g}_{1}=g_{3}+b_{0} u+\lambda_{1} g_{2},
$$

where $\lambda_{1}>0$ is the control parameter. 

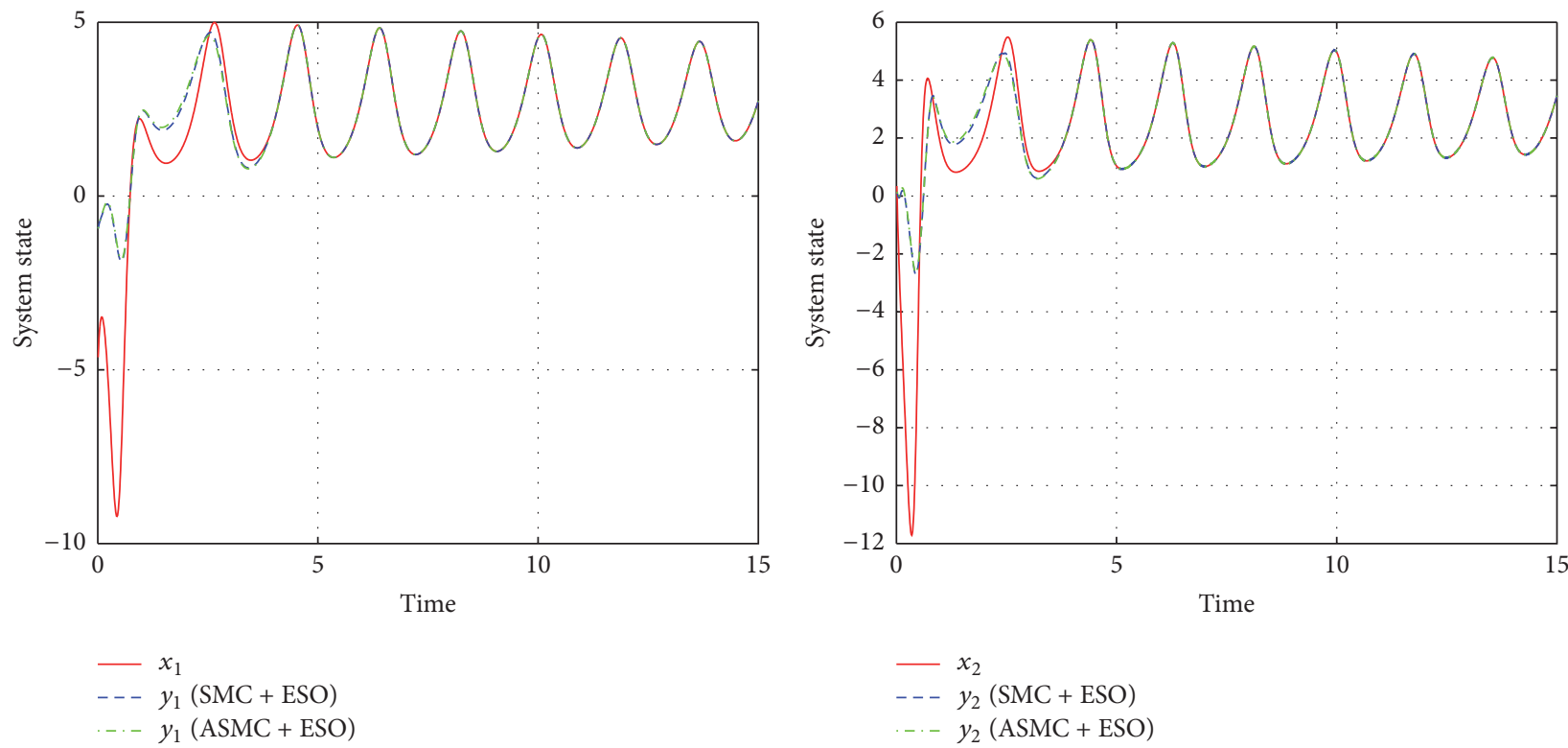

(a) Synchronization of $x_{1}, y_{1}$ for SMC + ESO and ASMC + ESO

(b) Synchronization of $x_{2}, y_{2}$ for SMC + ESO and ASMC + ESO

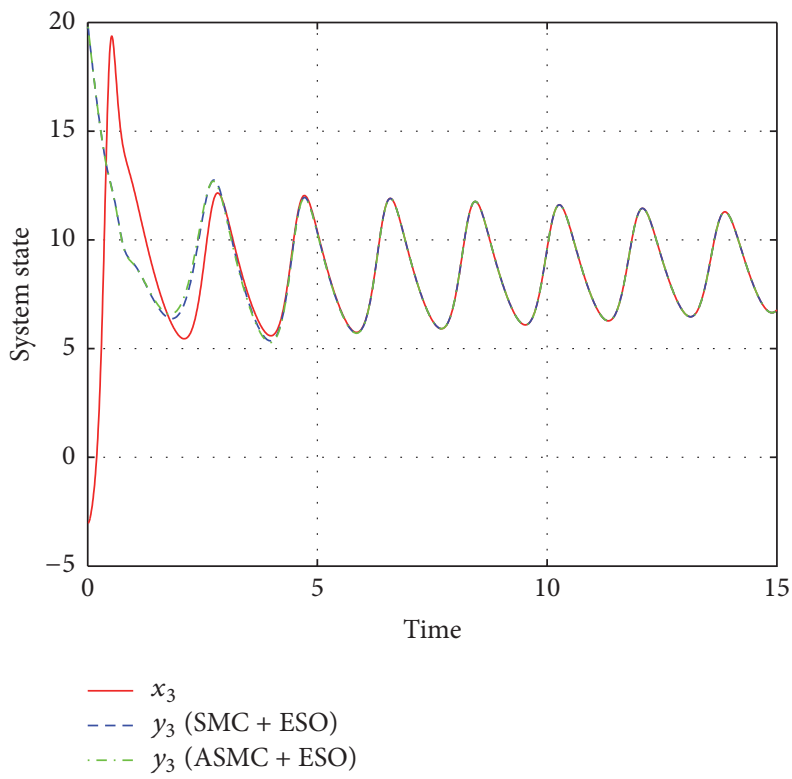

(c) Synchronization of $x_{3}, y_{3}$ for SMC + ESO and ASMC + ESO

FIGURE 1: Synchronization performance of the system states in Case 1.

According to (15) and (18), the traditional sliding mode controller using extended state observer (SMC + ESO) depicted in [17] is given by

$$
u^{*}=\frac{1}{b_{0}}\left(-z_{3}-\lambda_{1} z_{2}-k^{*} \operatorname{sign}(s)\right),
$$

where $k^{*}>0$ is a constant satisfying the condition that $k^{*} \geq d_{3}+\lambda_{1} d_{2}$, in which $d_{2}$ and $d_{3}$ are the upper bounds of estimation error.

Unfortunately, the control gain $k^{*}$ cannot be obtained accurately since the upper bounds $d_{2}$ and $d_{3}$ are difficult to be measured. This may lead to a negative influence on the system control performance. To solve the problem, an adaptive sliding mode controller using extended state observer (ASMC + ESO) is developed with the following expression:

$$
u=\frac{1}{b_{0}}\left(-z_{3}-\lambda_{1} z_{2}-k \operatorname{sign}(s)\right),
$$

where $k=k(t)$ is the adaptive control parameter designed as

$$
\dot{k}= \begin{cases}k_{m}|s| \operatorname{sign}(|s|-\epsilon) & k>\mu \\ \mu & k \leq \mu\end{cases}
$$



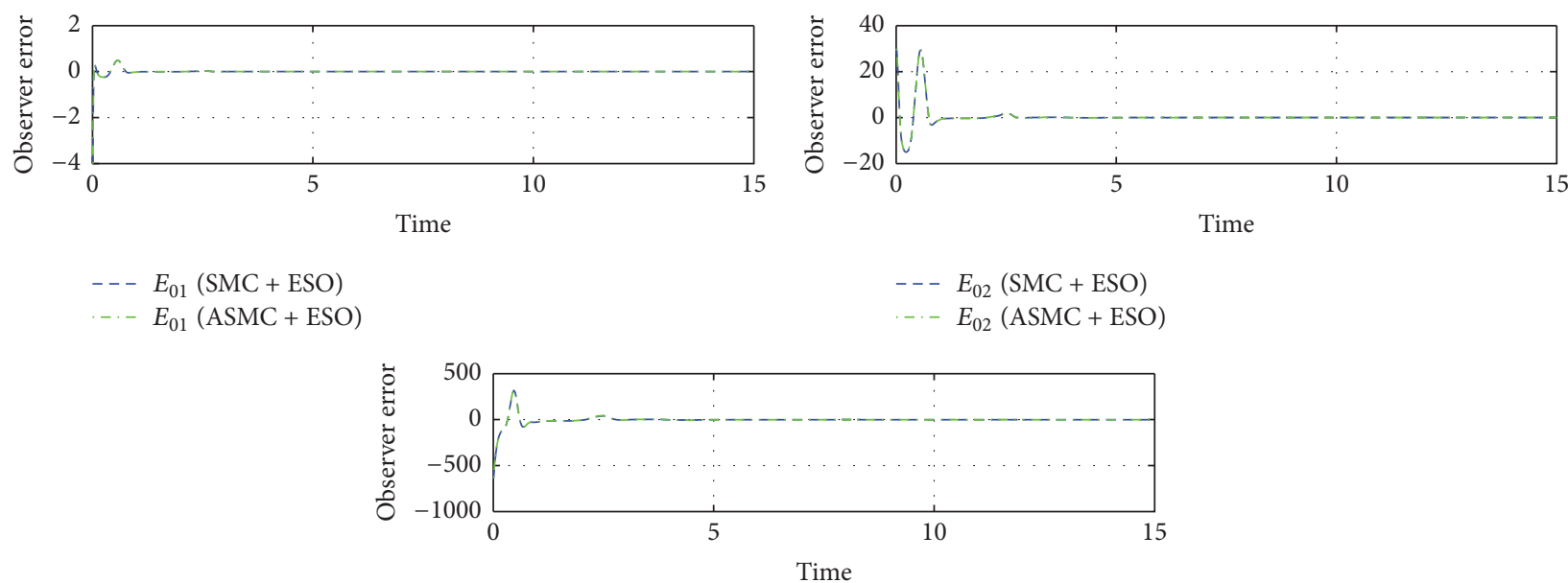

$--E_{03}(\mathrm{SMC}+\mathrm{ESO})$

-.- $E_{03}(\mathrm{ASMC}+\mathrm{ESO})$

Figure 2: Observer errors of ESO in Case 1.

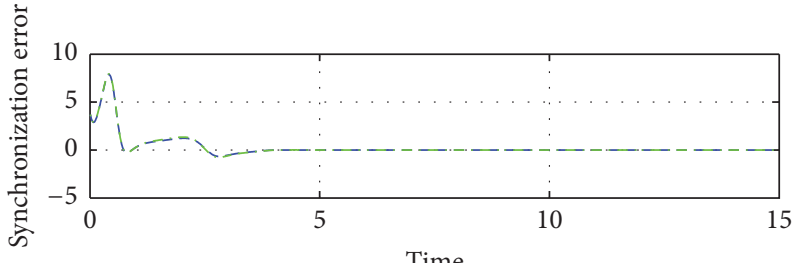

Time

$---e_{1}(\mathrm{SMC}+\mathrm{ESO})$

$e_{1}(\mathrm{ASMC}+\mathrm{ESO})$

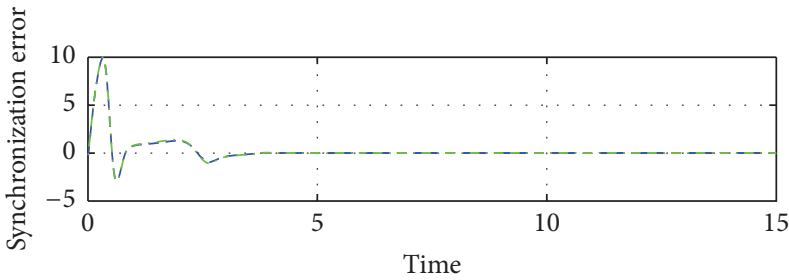

$---e_{02}(\mathrm{SMC}+\mathrm{ESO})$

$e_{02}(\mathrm{ASMC}+\mathrm{ESO})$

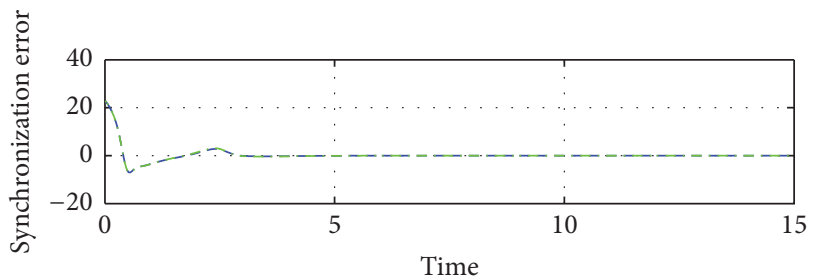

$---e_{3}(\mathrm{SMC}+\mathrm{ESO})$

$\cdots e_{3}(\mathrm{ASMC}+\mathrm{ESO})$

Figure 3: Synchronization errors in Case 1.

with $k_{m}$, $\epsilon$ and $\mu$ being small positive constants and used to guarantee $k>0$.

4.2. Stability Analysis. Before the system stability analysis, the following two lemmas are introduced.

Lemma 2 (see [18]). The parameter $k(t)$ has an upper bound in the nonlinear uncertain system (13) with the sliding mode surface (17); namely, there exists a desired value $k^{*}>0$ which can guarantee that $k(t) \leq k^{*}, \forall t>0$.

Lemma 3 (see [19]). Suppose there is a continuous positive definite function $V(t)$ which satisfies the following differential function:

$$
\dot{V}(t) \leq-\alpha V^{\eta}(t) \quad \forall t>0, V\left(t_{0}\right)>0,
$$

where $\alpha>0$ and $0<\eta<1$ are constants. Then, there exists a finite-time $t_{1}$ for the given time $t_{0}$, and we have the following inequality and equality relationships:

$$
\begin{aligned}
V^{1-\eta}(t) & \leq V^{1-\eta}\left(t_{0}\right)-\alpha(1-\eta)\left(t-t_{0}\right) \quad t_{0} \leq t \leq t_{1} \\
V(t) & \equiv 0, \quad \forall t \geq t_{1},
\end{aligned}
$$

where $t_{1}=t_{0}+V^{1-\eta}\left(t_{0}\right) / \alpha(1-\eta)$.

Theorem 4. Considering the uncertain PMSM subsystem (13), the sliding mode surface (17), the controller (20), and the parameter adaptive law (21), the sliding surface san converge to zero within a finite time. 


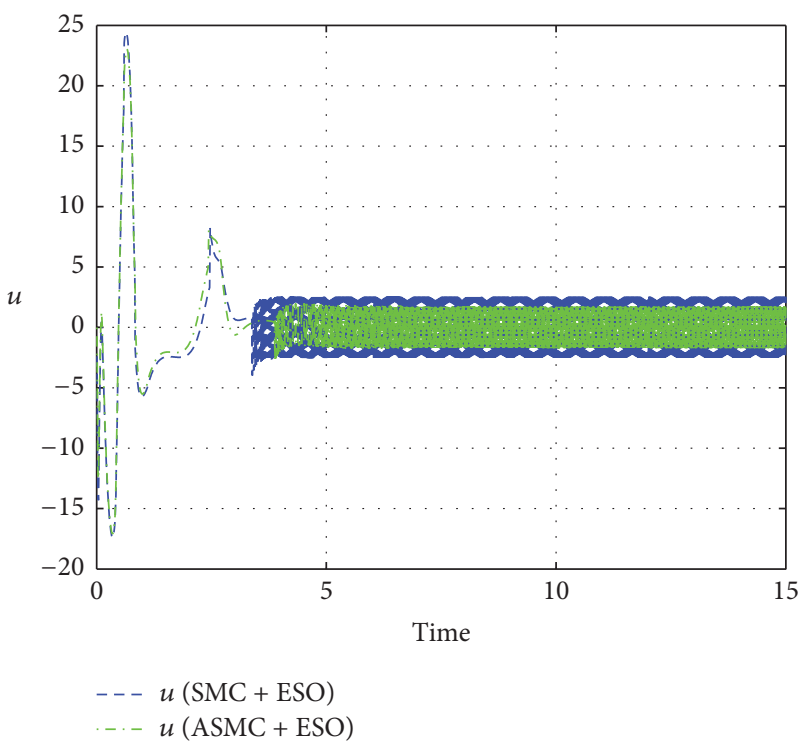

FIgURE 4: Control signals in Case 1.

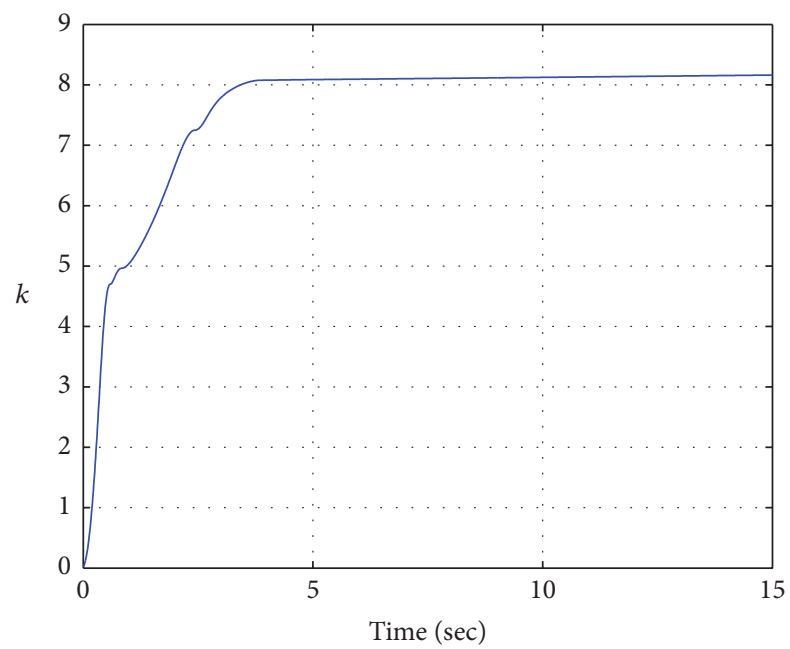

Figure 5: Adaptive parameter $k(t)$ in Case 1.

Proof. Define a Lyapunov function for system (13):

$$
V=\frac{1}{2} s^{2}+\frac{1}{2 \beta} \widetilde{k}^{2},
$$

where $\widetilde{k}=k-k^{*}$.

Differentiating $V$, we have

$$
\dot{V}=s \dot{s}+\frac{1}{\beta} \widetilde{k} \dot{\tilde{k}}=s\left(g_{3}+b_{0} u+\lambda_{1} g_{2}\right)+\frac{1}{\beta}\left(k-k^{*}\right) \dot{k} .
$$

Substitute (20) into (25), and we can obtain

$$
\begin{aligned}
\dot{V}= & s\left[\left(g_{3}-z_{3}\right)+\lambda_{1}\left(g_{2}-z_{2}\right)-k \operatorname{sign}(s)\right] \\
& +\frac{1}{\beta}\left(k-k^{*}\right) \dot{k}
\end{aligned}
$$

$$
\begin{aligned}
\leq & |s|\left(\left|g_{3}-z_{3}\right|+\lambda_{1}\left|g_{2}-z_{2}\right|\right)-k|s|+k^{*}|s| \\
& -k^{*}|s|+\frac{1}{\beta}\left(k-k^{*}\right) \dot{k} \\
\leq & -\left[k^{*}-\left(d_{3}+\lambda_{1} d_{2}\right)\right]|s|-\left(k-k^{*}\right)|s| \\
& +\frac{1}{\beta}\left(k-k^{*}\right) \dot{k} \\
= & -\left[k^{*}-\left(d_{3}+\lambda_{1} d_{2}\right)\right]|s|+\left(k-k^{*}\right)\left(\frac{1}{\beta} \dot{k}-|s|\right) .
\end{aligned}
$$

Introducing a new parameter $\alpha_{k}>0,(26)$ can be written as

$$
\begin{aligned}
\dot{V} \leq & -\left[k^{*}-\left(d_{3}+\lambda_{1} d_{2}\right)\right]|s|-\left(k-k^{*}\right)\left(|s|-\frac{1}{\beta} \dot{k}\right) \\
& +\alpha_{k}\left|k-k^{*}\right|-\alpha_{k}\left|k-k^{*}\right| .
\end{aligned}
$$

According to Lemma 2 and (27), it can be concluded that

$$
\begin{aligned}
\dot{V} & \leq-\alpha_{d}|s|-\xi-\alpha_{k}\left|k-k^{*}\right| \\
& =-\sqrt{2} \alpha_{d} \frac{|s|}{\sqrt{2}}-\sqrt{2 \beta} \alpha_{k} \frac{\left|k-k^{*}\right|}{\sqrt{2 \beta}}-\xi \\
& \leq-\alpha_{m} V^{1 / 2}-\xi
\end{aligned}
$$

where $\alpha_{d}=k^{*}-\left(d_{3}+\lambda_{1} d_{2}\right)>0, \xi=\left((1 / \beta) \dot{k}-|s|-\alpha_{k}\right)\left|k-k^{*}\right|$, and $\alpha_{m}=\min \left\{\sqrt{2} \alpha_{d}, \sqrt{2 \beta} \alpha_{k}\right\}$.

Since $\mu$ and $\varepsilon$ are both small constants, without loss of generality, we only discuss the situation for $k>\mu$. When $k>$ $\mu$, two different cases are discussed according to relationship between $|s|$ and $\epsilon$.

(a) When $|s|>\epsilon,(21)$ can be rewritten as $\dot{k}=k_{m}|s|, \xi=$ $\left((1 / \beta) k_{m}|s|-|s|-\alpha_{k}\right)\left|k-k^{*}\right|$. Choosing $\beta<k_{m} \epsilon /\left(\alpha_{k}+\epsilon\right)$, we can conclude $\xi>0$.

(b) When $|s| \leq \epsilon$, we have $\dot{k}=-k_{m}|s|$, it can be concluded that $\xi<0, \dot{k}<0$, and $k$ is gradually decreasing. Thus, we can obtain $\dot{s}>0$ when $k$ is reduced to $0 \leq k \leq d_{3}+\lambda_{1} d_{2}$. Consequently, sliding mode $s$ will increase and achieve to the range of $|s|>\epsilon$.

Similarly, we can guarantee $\xi>0$ by choosing the appropriate parameter $\beta$ when $k>\mu$. Then, from (28) and the above discussion, we have $\dot{V} \leq-\alpha_{m} V^{1 / 2}$. According to Lemma 3 , there exists a finite-time $t_{1}$ satisfying $V(t) \equiv 0$ as $t \geq t_{1}$. This can ensure the convergence of the sliding mode $s$ within a finite time.

Theorem 5. The state variables $e_{1}, e_{2}, e_{3}$ in error system (8) will converge to zero when the states $g_{1}, g_{2}$ in system (13) achieve the sliding surface $s=0$.

Proof. System (13) have invariant characteristics when the states $g_{1}, g_{2}$ achieve $s=0$, and from (14) and (17) we have $\dot{g}_{1}+\lambda_{1} g_{1}=0$. Then, solving the first-order differential function, we can obtain $g_{1}=e^{-\lambda_{1} t}$, which means that the 


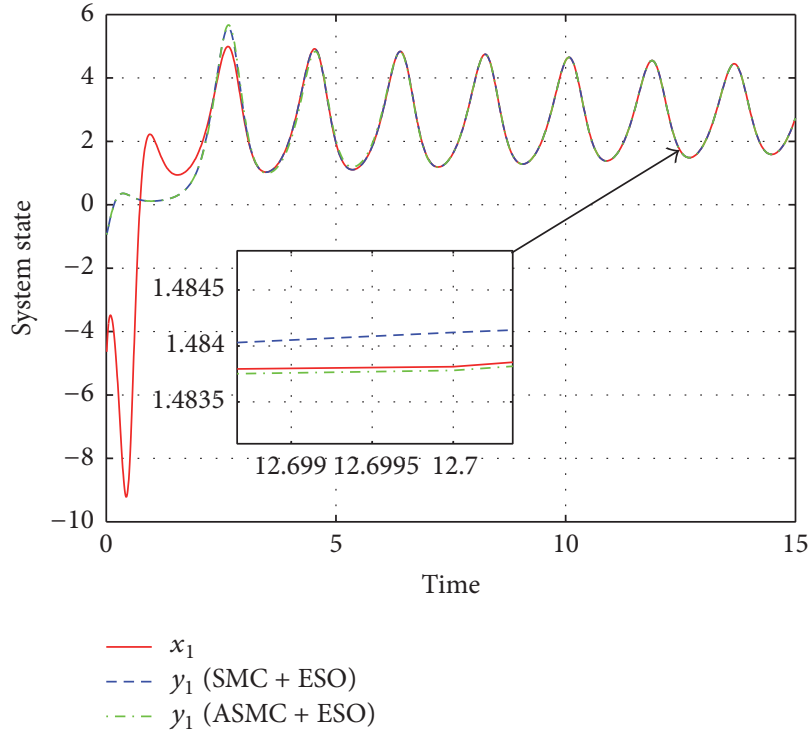

(a) Synchronization of $x_{1}, y_{1}$ for SMC + ESO and ASMC + ESO

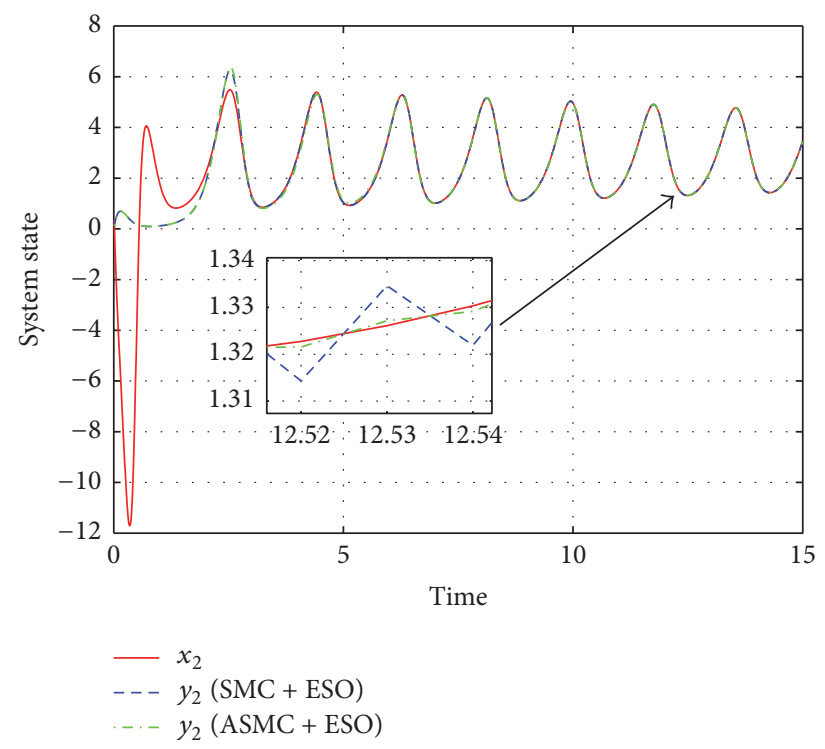

(b) Synchronization of $x_{2}, y_{2}$ for SMC + ESO and ASMC + ESO

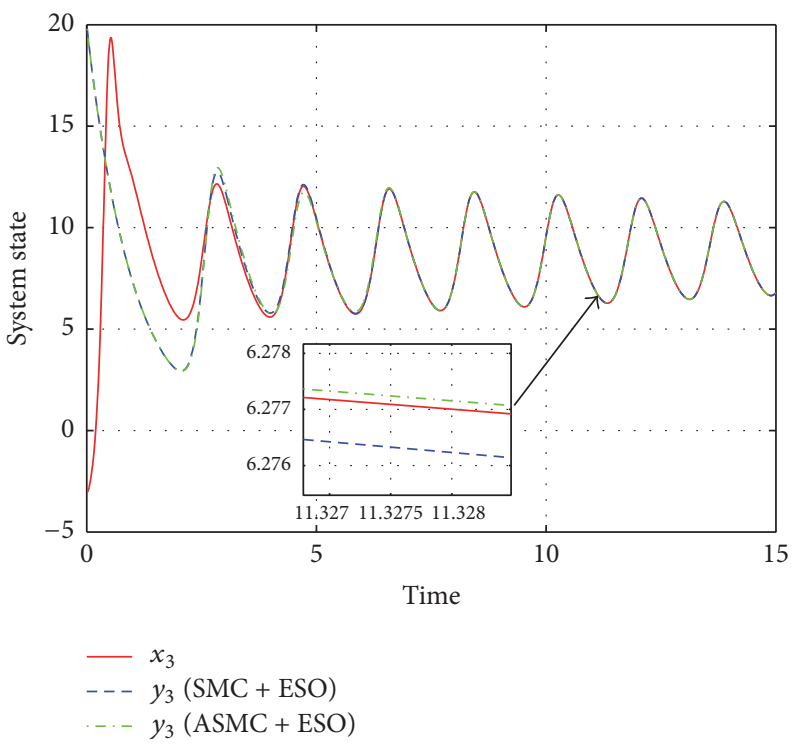

(c) Synchronization of $x_{3}, y_{3}$ for SMC + ESO and ASMC + ESO

FIGURE 6: Synchronization performance of the system states in Case 2.

state $g_{1}$ can converge to zero when time tends to infinity. Also, according to (17), we have the similar result that $g_{2}$ will converge to zero when time tends to infinity.

Consequently, the state variables $e_{1}, e_{2}, e_{3}$ in error system (8) have the following relationship according to (11) and (12): $e_{1}, e_{2}$ converge to zero and $\dot{e}_{3}=-e_{3}$ when time tends to infinity. Thus, we can conclude that $e_{1}, e_{2}, e_{3}$ will asymptotically stabilized to zero. This completes the proof.

\section{Simulation}

In order to verify the effectiveness of the proposed method, a traditional sliding mode control based on extended state observer $(\mathrm{SMC}+\mathrm{ESO})$ is adopted to compare with the proposed adaptive control method (ASMC + ESO). The initial conditions and parameters in the simulations are set the same for a fair comparison; that is, the sampling time is set as $T_{s}=0.01$; the initial conditions are given as $\left(x_{1}(0), x_{2}(0), x_{3}(0)\right)=(-5,1,-3),\left(y_{1}(0), y_{2}(0), y_{3}(0)\right)=$ $-1,0.01,20$; the parameters of sliding mode control and extended state observer are chosen as $\lambda_{1}=10, b_{0}=5$, $\beta_{1}=60, \beta_{2}=200, \beta_{3}=0.01, \alpha_{1}=0.5, \alpha_{2}=0.25, \alpha_{3}=0.125$, $\delta=0.01$, and $\sigma=5.46$; the control parameter in SMC + ESO is given by $k^{*}=12$; the control parameters in ASMC + ESO are set as $k_{m}=0.15, \epsilon=0.01$, and $\mu=0.0001$.

Case 1 (the controller $u$ works at initial time $t=0$ ). The simulation results are shown in Figures 1-5. Figure 1 provides 

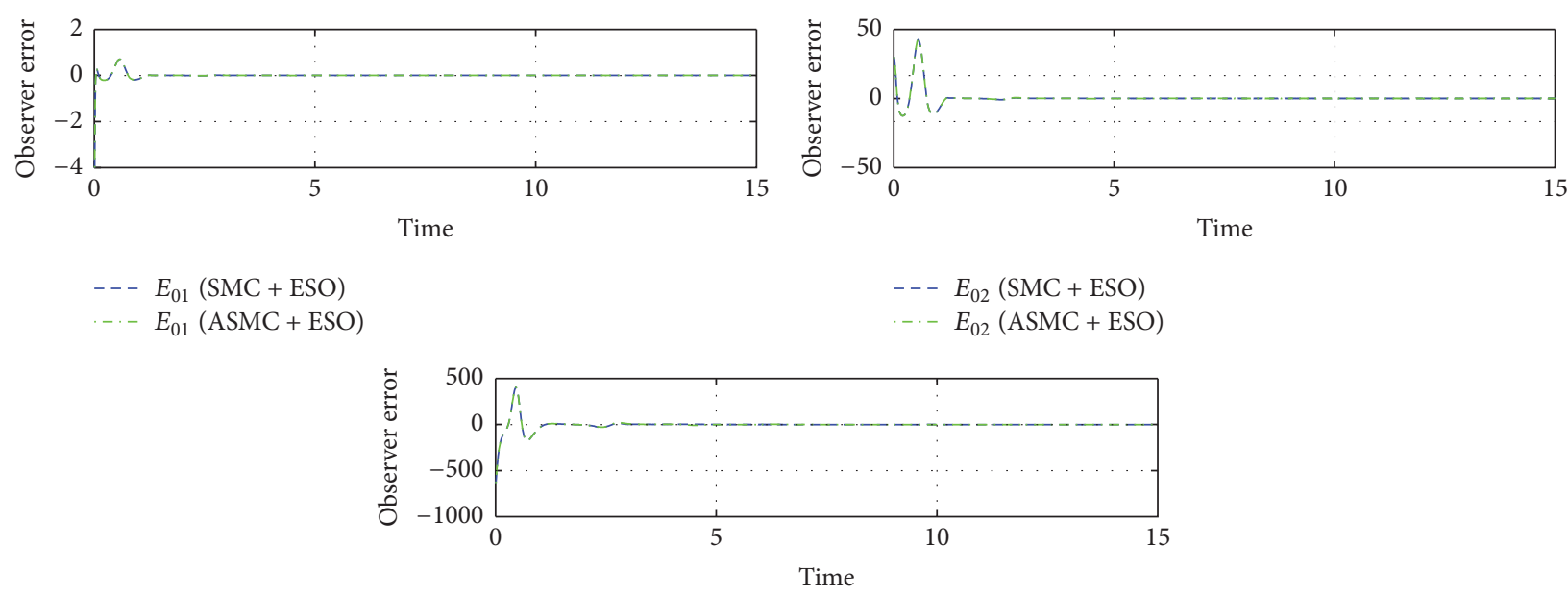

$--E_{03}(\mathrm{SMC}+\mathrm{ESO})$

$-E_{03}(\mathrm{ASMC}+\mathrm{ESO})$

Figure 7: Observer errors of ESO in Case 2.

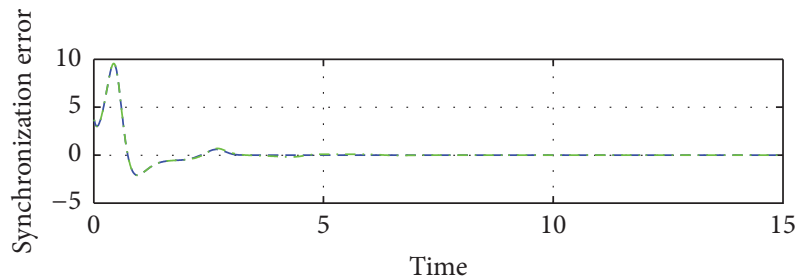

Time

$--e_{1}(\mathrm{SMC}+\mathrm{ESO})$

$e_{1}(\mathrm{ASMC}+\mathrm{ESO})$

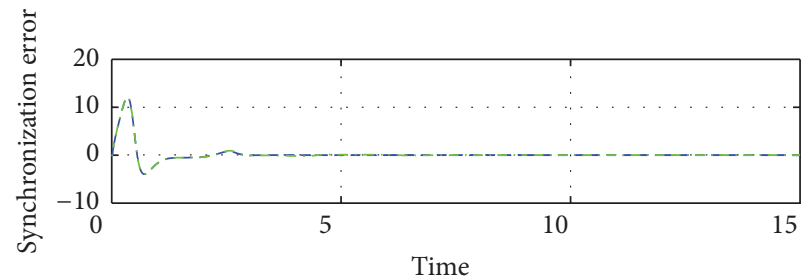

$---e_{02}(\mathrm{SMC}+\mathrm{ESO})$

$\cdots e_{02}(\mathrm{ASMC}+\mathrm{ESO})$

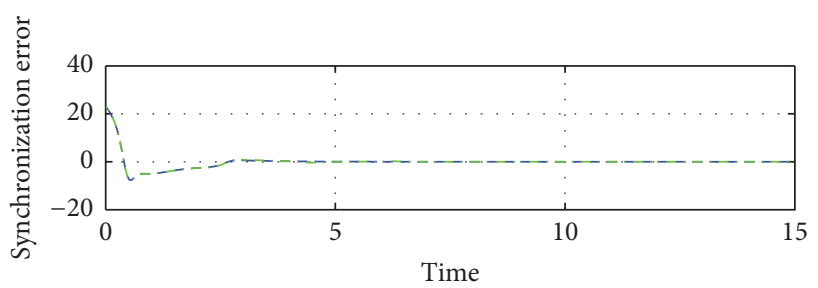

$---e_{3}(\mathrm{SMC}+\mathrm{ESO})$

$\cdots-e_{3}(\mathrm{ASMC}+\mathrm{ESO})$

FIGURE 8: Synchronization errors in Case 2.

the synchronization performance of the system states. The observer errors of ESO and synchronization errors are shown in Figures 2 and 3, respectively. The control signal is given by Figure 4. As shown in Figures 1-3, the compared two control methods, that is, SMC + ESO and ASMC + ESO, can both achieve satisfactory chaos synchronization control performance; the observer errors of ESO and system synchronization errors can rabidly converge to zero. However, from Figure 4 we can see that the amplitude of ASMC + ESO is smaller than SMC + ESO, and the chattering phenomenon of control signal in ASMC + ESO is also smaller when the system is stable. The adaptation curve of the parameter $k(t)$ is shown in Figure 5. As can be seen from Figure 5, the parameter $k(t)$ converges to 8.2 , which is slightly less than the parameter $k^{*}$ in SMC + ESO.
Case 2 (the controller $u$ works at $t=2 s$ ). The parameters and initial conditions are all the same as those in Case 1 for fair comparison. The synchronization performance of the system states, observer errors of ESO, synchronization errors, control signals, and the adaptive parameter $k(t)$ are shown in Figures 6-10, respectively. As can be seen from Figures $6-8$, the control input is delayed to be working by 2 seconds, and the error system is not well controlled by using SMC + ESO; however, the ASMC + ESO can still have a good chaos synchronization control performance after a slight chattering. From Figure 9, we can clearly see that the chattering phenomenon of the control signal in SMC + ESO is significantly larger than that of ASMC + ESO. The reason is that, for the fixed parameter $k$ in SMC + ESO, the condition that $k^{*} \geq d_{3}+\lambda_{1} d_{2}$ may not be always satisfied any 


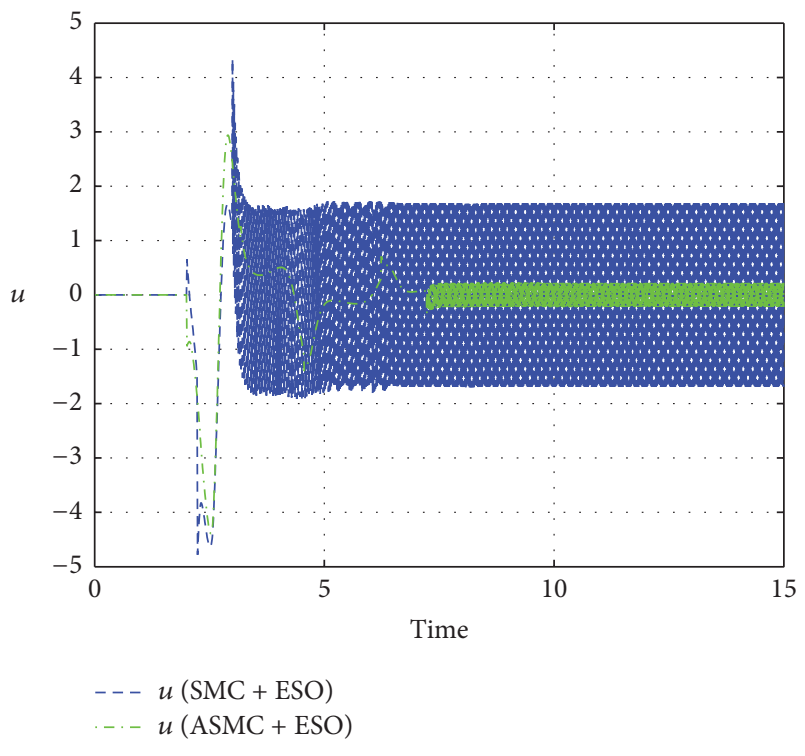

FIGURE 9: Control signals in Case 2.

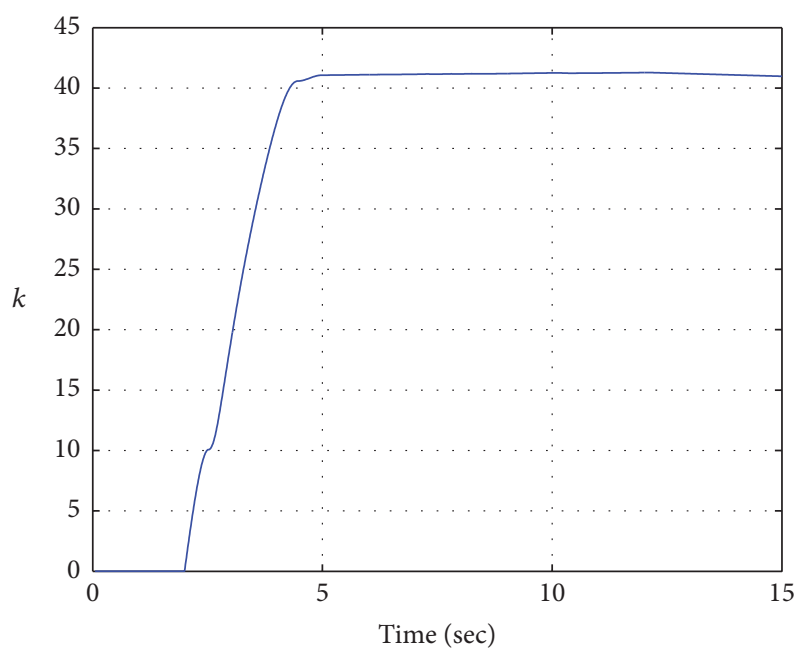

Figure 10: Adaptive parameter $k(t)$ in Case 2.

more, but the control parameter $k(t)$ in ASMC + ESO is an adaptive parameter, which can always satisfy the condition. From Figure 10, it can be seen that the parameter $k(t)$ will converge to 42 , which is much larger than the setting value $k^{*}=12$ in SMC + ESO.

\section{Conclusion}

In this paper, an adaptive sliding mode control method using extended state observer is presented to guarantee the synchronization control performance for two chaotic PMSM systems. An adaptive parameter is designed for the control gain to improve the suitability for different control situation and reduce the chattering in the control signal, and the extended state observer is adopted to estimate the system uncertainties. The simulation results indicate that the system can achieve a good synchronization control performance for different initial conditions.

\section{Competing Interests}

The authors declare that they have no competing interests.

\section{Acknowledgments}

This work was supported by the Major Research Plan of the National Natural Science Foundation of China (Grants nos. 91438117 and 91538202) and National Natural Science Foundation of China (Grant no. 61403343).

\section{References}

[1] I. Ahmad, A. Bin Saaban, A. B. Ibrahim, and M. Shahzad, "Global chaos synchronization of new chaotic system using linear active control," Complexity, vol. 21, no. 1, pp. 379-386, 2015.

[2] Q. Chen, X. M. Ren, and J. Na, "Robust finite-time chaos synchronization of uncertain permanent magnet synchronous motors," ISA Transactions, vol. 58, no. 1, pp. 262-269, 2015.

[3] W. L. Ditto and S. Sinha, "Exploiting chaos for applications," Chaos, vol. 25, no. 9, Article ID 097615, 2015.

[4] J. Guan, "Adaptive modified generalized function projection synchronization between integer-order and fractional-order chaotic systems," Optik, vol. 127, no. 10, pp. 4211-4216, 2016.

[5] J. K. Wang, X. Q. Chen, and J. K. Fu, "Adaptive finite-time control of chaos in permanent magnet synchronous motor with uncertain parameters," Nonlinear Dynamics, vol. 78, no. 2, pp. 1321-1328, 2014.

[6] Y.-Y.Hou, "Finite-time chaos suppression of permanent magnet synchronous motor systems," Entropy, vol. 16, no. 4, pp. 22342243, 2014.

[7] K. L. Su and C. L. Li, "Chaos control of permanent magnet synchronous motors via unidirectional correlation," Optik, vol. 125, no. 14, pp. 3693-3696, 2014.

[8] T.-B.-T. Nguyen, T.-L. Liao, and J.-J. Yan, "Adaptive sliding mode control of chaos in permanent magnet synchronous motor via fuzzy neural networks," Mathematical Problems in Engineering, vol. 2014, Article ID 868415, 11 pages, 2014.

[9] X. H. Yang, X. P. Liu, L. L. Hu et al., "Robust sliding mode variable structure synchronization control of chaos in permanent magnet synchronous motor," Modular Machine Tool and Automatic Manufacturing Technique, vol. 1, no. 8, pp. 93-98, 2012.

[10] Q. Chen, Y.-R. Nan, H.-H. Zheng, and X.-M. Ren, "Full-order sliding mode control of uncertain chaos in a permanent magnet synchronous motor based on a fuzzy extended state observer," Chinese Physics B, vol. 24, no. 11, Article ID 110504, 2015.

[11] X. H. Yang, X. P. Liu, H. S. Liu et al., "Fuzzy sliding-mode control in permanent magnet synchronous motor," International Journal of Digital Content Technology and its Applications, vol. 7, no. 1, pp. 526-535, 2013.

[12] A. Loria, "Robust linear control of (chaotic) permanent-magnet synchronous motors with uncertainties," IEEE Transactions on Circuits and Systems I: Regular Papers, vol. 56, no. 9, pp. 21092122, 2009. 
[13] S. H. Luo, "Adaptive fuzzy dynamic surface control for the chaotic permanent magnet synchronous motor using Nussbaum gain," Chaos, vol. 24, no. 3, Article ID 033135, pp. 58805885, 2014.

[14] S. Luo, "Nonlinear dynamic surface control of chaos in permanent magnet synchronous motor based on the minimum weights of RBF neural network," Abstract and Applied Analysis, vol. 2014, Article ID 609340, 9 pages, 2014.

[15] Q. Wei, X.-Y. Wang, and X.-P. Hu, "Optimal control for permanent magnet synchronous motor," Journal of Vibration and Control, vol. 20, no. 8, pp. 1176-1184, 2014.

[16] J. Na, Q. Chen, X. M. Ren, and Y. Guo, "Adaptive prescribed performance motion control of servo mechanisms with friction compensation," IEEE Transactions on Industrial Electronics, vol. 61, no. 1, pp. 486-494, 2014.

[17] S. Li, M. Zhou, and X. Yu, "Design and implementation of terminal sliding mode control method for PMSM speed regulation system," IEEE Transactions on Industrial Informatics, vol. 9, no. 4, pp. 1879-1891, 2013.

[18] S. Yu, X. H. Yu, B. Shirinzadeh, and Z. Man, "Continuous finitetime control for robotic manipulators with terminal sliding mode," Automatica, vol. 41, no. 11, pp. 1957-1964, 2005.

[19] Q. Chen, L. Yu, and Y. Nan, "Finite-time tracking control for motor servo systems with unknown dead-zones," Journal of Systems Science \& Complexity, vol. 26, no. 6, pp. 940-956, 2013. 


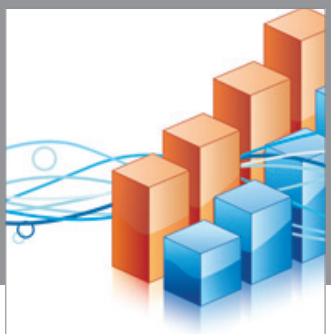

Advances in

Operations Research

vatem alat4

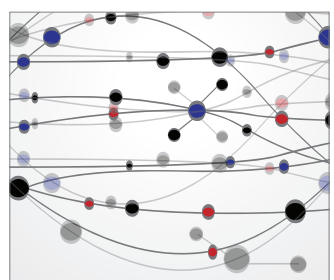

\section{The Scientific} World Journal
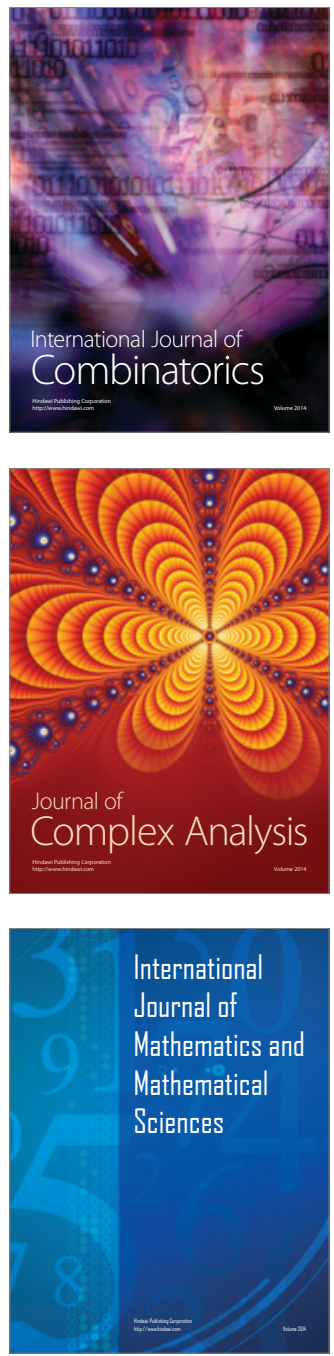
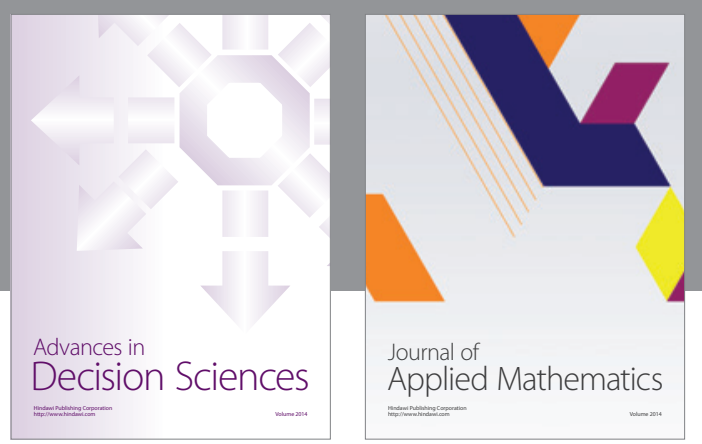

Algebra

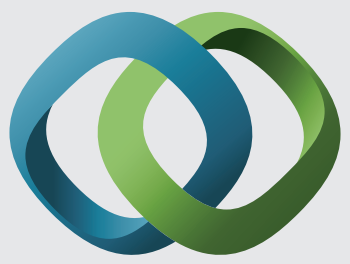

\section{Hindawi}

Submit your manuscripts at

http://www.hindawi.com
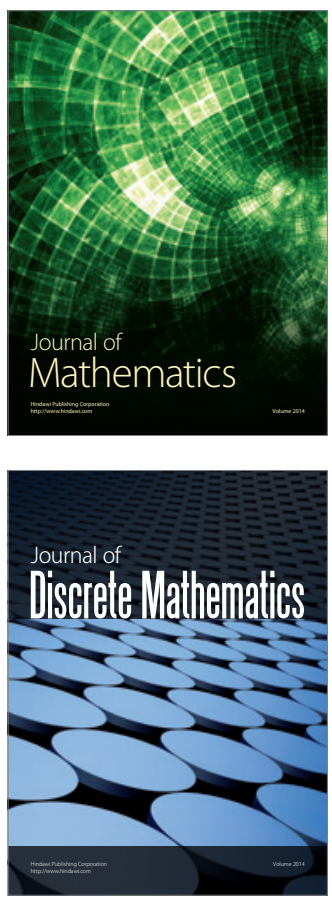

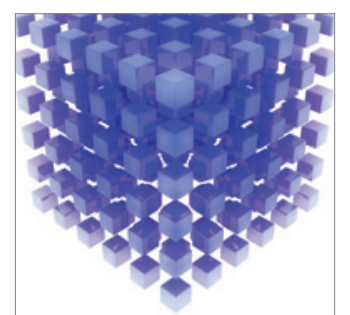

Mathematical Problems in Engineering
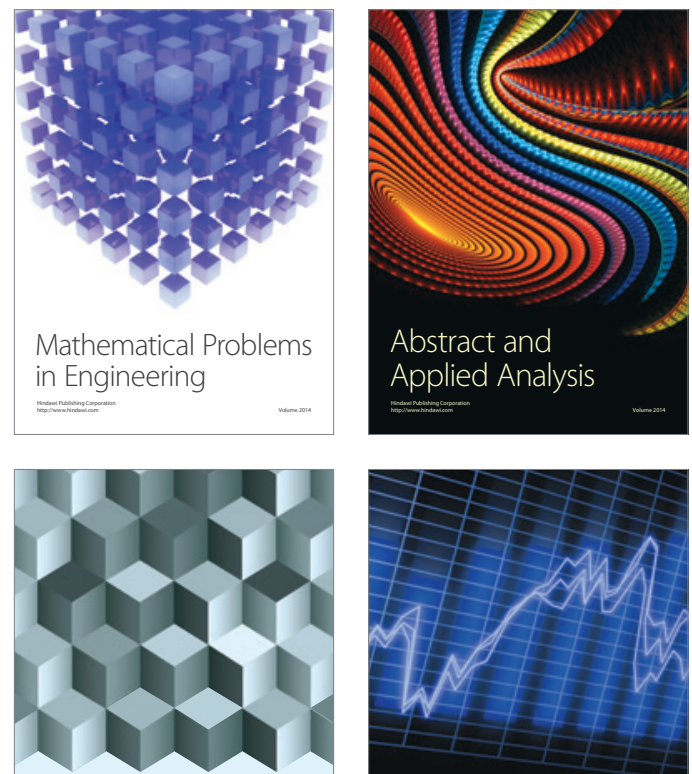

Journal of

Function Spaces

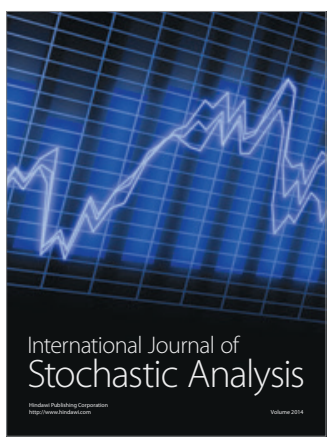

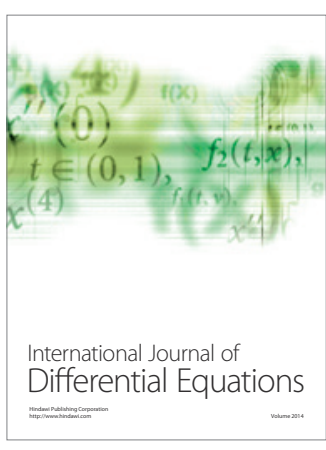
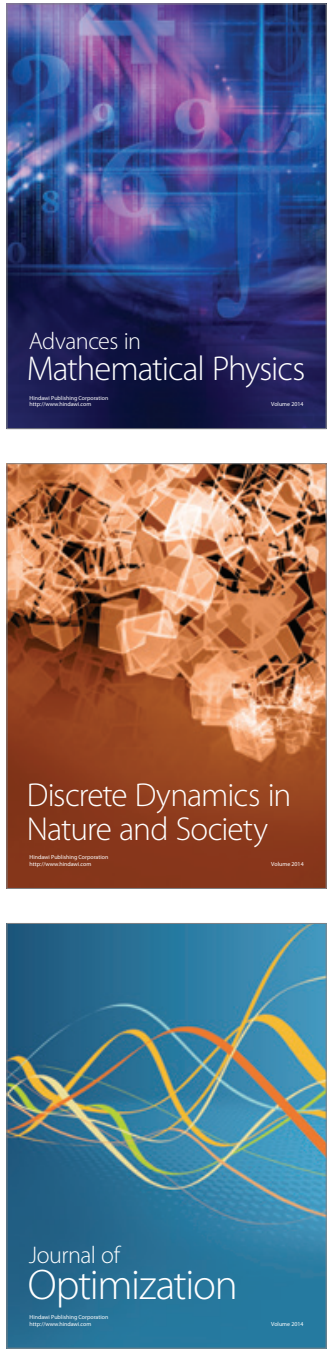